\title{
perifèria
}

Número 5, Diciembre 2006

www.periferia. name

\section{Etnografía, brujería y tensión social: las tribulaciones de Marwick para poner a prueba la tesis obstétrica matrilineal}

\author{
Aurora González Echevarría - UAB - Grup de recerca sobre investigació \\ fonamental i orientada (GRAFO) ${ }^{1}$
}

\section{Resumen}

El intento frustado de Marwick para poner a prueba una hipótesis sobre las causas de la brujería con los datos etnográficos recogidos de forma sistemática sobre los cewa pone de manifiesto la necesidad de realizar diseños de investigación específicos que incluyan los indicadores necesarios para que esta contrastación pueda llevarse a cabo de forma efectiva. De esta forma la investigación etnográfica debe ser emergente, pero solamente en el sentido de permitir rediseños sucesivos para la contrastación de las hipótesis que surgen en el trabajo de campo.

\begin{abstract}
The Marwick failed attempt to test one hypothesis about the causes of sorcery with the ethnographic data collected in a systematic way about the cewa shows how specific research designs should be made in order to test specific hypothesis with its own proxies. In this way, the ethnographic research should be emergent but only in the sense to allow successive research designs for effective testing of emergent hypothesis during the fieldwork.
\end{abstract}

En la segunda mitad del siglo XX apuntó en Antropología una efímera revolución metodológica. Cuando la traducción al inglés de Popper extendió sus tesis sobre la irrelevancia del proceso que llevaba a la formulación de hipótesis y cuanto más tarde Feyerabend sostuvo que el único método era el de usar todos los métodos, pidiendo que en la formulación de hipótesis no se suprimieran ni siquiera los procedimientos más extravagantes del cerebro humano, que se permitiera "el conflicto, la flexibilidad individual, una suave locura" (1974: 162,42), algunos antropólogos se sintieron liberados del corsé empirista. Entre ellos Jarvie, que defendió la imaginación frente a los hechos:

\footnotetext{
${ }^{1}$ Enviar correspondencia a: aurora.gonzalez@uab.es
} 


\section{perifèria}

Número 5, Diciembre 2006

www.periferia. name

La cuestión no es inhibir nuestra imaginación, sino ser muy crítico respecto a sus productos; tal vez el científico debería estar borracho la noche antes y sobrio como una piedra la mañana siguiente (1967: 6).

De Jarvie escribió Gellner, en el prólogo a The Revolution in Anthropology, que había elegido para su libro un título deliberadamente ambiguo porque, al tiempo que describe una revolución que sucedió en la generación anterior -la revolución de Malinowski-, está ansioso por encabezar otra, antiinductivista, que estaba a punto de producirse.

Se trataba de una revolución contra el trabajo de campo, o, más exactamente, contra su escasa productividad teórica, centrada en la concepción simplificada del inductivismo que informó el empirismo antropológico de la primera mitad del siglo. En la ciencia moderna no se defendió nunca un método inductivo estricto que se detuviera cuando se llegaba a explicar los fenómenos particulares en términos de leyes generales. A la confianza en un conocimiento que fuera de lo particular a lo general, se añadió la exigencia de corroboración adicional para las explicaciones generales. De ahí el énfasis en la experimentación y el avance real del conocimiento. Pero la Antropología, cuando se declaró inductivista, combinó la defensa de un método de inducción-deducción-contrastación adicional con una práctica científica deficiente, que se detuvo en principios o explicaciones que suponía generales sin llegar a la puesta a prueba de los principios ni de las explicaciones.

Como causas de esta distorsión metodológica se señalaron tanto la urgencia por recoger información sobre el mayor número posible de pueblos y culturas distintos que estaban desapareciendo o transformándose, como en la ilusión holista. En efecto, sólo el ideal de que los datos recogidos debían ser de toda la cultura pudo hacer pensar que sería posible más adelante formular teorías y contrastarlas. Así, cuando Meyer Fortes, en 1951, hizo un balance de los principales logros de la Antropología Social británica, destacó la acumulación de datos obtenidos por observadores bien preparados, porque pensaba que iban a proporcionar más 


\section{perifèria}

Número 5, Diciembre 2006

www.periferia. name

oportunidades que nunca para que los antropólogos "de sillón" construyeran y pusieran a prueba hipótesis con la ayuda de información detallada y fiable (Fortes, 1975: 170). Irónicamente, sólo seis años más tarde Murdock, 1957, cuestionaría la prevalencia de la grupos de filiación unilineal en las sociedades tradicionales, con las consecuencias de todos conocidas sobre el principio teórico fundamental de la antropología británica, el principio de filiación. Pero Fortes confiaba en un desarrollo espectacular de la teoría de la filiación porque confiaba en que las monografías acumuladas eran, de acuerdo con el ideal, holistas.

Poco tiempo después, la defensa de la utilización exclusiva de metodologías hermenéuticas en Antropología, en la estela de Winch (1958) y de Gadamer (1960) arrinconó la discusión sobre métodos inductivos o deductivos, erróneamente clasificados como "positivismo". Casi 50 años más tarde, tenemos tan pocas razones para basarnos sólo en Popper como para basarnos sólo en Gadamer, pero subsiste el problema de la puesta a prueba. El énfasis en que el trabajo de campo no empieza con hipótesis sino con preguntas y el énfasis en que no termina con explicaciones, sino con interpretaciones no nos libera de la tarea fundamental de validar hasta donde podamos nuestras conclusiones, 0 , si se prefiere, de las respuestas a las preguntas que guían nuestra investigación que, pese a las connotaciones "positivistas", no pueden ser más que conjeturas o hipótesis. Aunque, como en Agar (1982), se prefiera hablar, tal vez para escapar a las connotaciones positivistas o cientifistas, de esquemas revisables. Me he ocupado de los usos del término "inductivismo" y de los usos del término "hermenéutica" en un artículo reciente, en el que contrapongo hermenéutica ontológica y procedimientos de interpretación y recojo la revisión de esquemas de Agar como excelente ejemplo de método para la puesta a prueba de hipótesis interpretativas. 


\section{perifèria}

Número 5, Diciembre 2006

www.periferia. name

Todo el que haya intentado poner a prueba una hipótesis transcultural con fuentes etnográficas preexistentes sabe que pocas veces encuentra los datos que busca. Fue una constatación de este tipo, buscando datos sobre ciertas prácticas adivinatorias, la que me haría interesarme primero por el valor de la etnografía acumulada para la formulación y puesta a prueba de las teorías antropológicas y de manera más general por los métodos de investigación en Antropología.

Pero la dificultad de puesta a prueba no acecha sólo a ingenuos estudiantes de antropología que preparan memorias de licenciatura en bibliotecas no siempre bien dotadas, también se cierne sobre etnógrafos que han vuelto a casa e intentan llegar a conclusiones sobre hipótesis o preguntas que no habían guiado su trabajo de campo. A juzgar por la literatura antropológica, la mayor parte de las veces de esta dificultad no son conscientes, y otras la ocultan. En varias publicaciones de los sesenta, Marwick ha tenido la honestidad de hablarnos de los problemas con que se encontró para la puesta a prueba de una hipótesis sobre los conflictos por la sucesión a la jefatura de los linajes matrilineales cewa y de cómo abandonó el problema sin resolverlo. Pienso que la causa residió en que ningún momento de la investigación se había diseñado para poner a prueba esta hipótesis. Y que las preguntas, como las respuestas hipotéticas, surgen antes, durante y después del trabajo de campo, muchas veces al escribir las conclusiones. Tanto las "quiebras" , en el sentido de Agar 1982, que se producen durante la investigación como los problemas y contradicciones que aparecen cuando se analizan los datos recogidos, son fuentes de hipótesis nuevas. Hipótesis que difícilmente pueden ponerse a prueba con los mismos datos que las han sugerido.

He recogido las tribulaciones de Marwick en dos textos anteriores (González Echevarría, 1984, 1987) pero ahora que los debates del GRAFO nos han hecho volver sobre dos textos de Teresa San Román (1983, 1996) sobre las distintas etapas del trabajo etnográfico y que en la UAB se pone en marcha un Master sobre investigación etnográfica y comparación transcultural, me ha parecido útil volver sobre ellas. Porque, como sucede con frecuencia, renombrar los problemas no los 


\section{perifèria}

Número 5, Diciembre 2006

www.periferia. name

resuelve y hablar en un diseño preliminar de cuales son las preguntas de investigación, invocar una metodología emergente o refugiarse en que las conclusiones no son más que interpretaciones no nos permite escapar a la validación de las respuestas, por tentativa que sea esta validación.

\section{El programa de investigación sobre la brujería africana}

Como sucede casi siempre, excepto en investigaciones que son completamente pioneras, el trabajo de Marwick se inscribe en lo que podríamos denominar, de acuerdo con el uso de Lakatos, un programa de investigación, en este caso el iniciado por Evans-Pritchard sobre el papel de los conflictos sociales en la génesis de acusaciones de brujería en sociedades tribales africanas.

Evans-Pritchard (1976 [1937]) había dedicado un capítulo a mostrar, en parte mediante unos cuantos ejemplos (pp. 118-119) que "quienes padecen desgracias buscan brujos entre sus enemigos". Como Mary Douglas (1976) ha defendido con detalle, tras la Segunda Guerra Mundial, de los caminos abiertos por el trabajo de Evans-Pritchard, no interesó tanto su aportación a la sociología del conocimiento como los aspectos políticos de las acusaciones de brujería.

Mónica Wilson, en 1950, puso de manifiesto que entre los nyakyusa las acusaciones de brujería típicas se hacían contra vecinos (de 38 casos de infortunio atribuidos a brujería que estudia, 23 se acusa a compañeros de poblado) (Wilson, 1970: 198214). En su opinión las causas habría que buscarlas en la estructura de los poblados de edad nyakyusa, en oposición a las reglas de herencia patrilineales. Como es sabido, los niños nyakyusa van construyendo progresivamente, junto con sus compañeros de edad, un poblado al que terminan por trasladarse y al que llevarán a sus esposas. Esto hace que, a diferencia de lo que sucede en la mayor parte de las comunidades locales del África subsahariana, no son las relaciones de parentesco las que marcan la moralidad, sino las normas de generosidad y hospitalidad entre vecinos. Y las acusaciones de brujería contribuirían al control de esta situación social (Wilson, 1951: 309 y ss.). Nadel, por su parte, ha observado que las acusaciones de brujería entre los nupe afectan principalmente a mujeres, y 


\section{perifèria}

Número 5, Diciembre 2006

www.periferia. name

lo atribuye a la hostilidad entre los hombres y las mujeres nupe, poco dependientes a causa de la libertad que les otorga su dedicación al comercio (Nadel, 1952 y 1970: 172-175). No voy a discutir ahora la adecuación de estas explicaciones, que me interesan sólo en este punto como ejemplo del interés por la función política de las acusaciones de brujería².

Un conjunto notable de acusados son, en algunas sociedades africanas, jefes de segmentos locales de un linaje o candidatos a serlo. Parece que en estos casos las acusaciones sirven para frenar o precipitar un proceso de fisión exigido por conflictos internos o por el crecimiento demográfico. Se ha dicho que en estas situaciones la potente ideología del parentesco que mantiene unido al linaje debe ser rota por una ideología igual de fuerte: la de la brujería. Para desobedecer al anciano lugbara hay que hacer de él un ser blanco, peligroso, caníbal, inclinado al incesto: más peligroso que los europeos o los azande, de los que ya todo se espera, el enemigo dentro, un ser social invertido en el núcleo de la organización social (Middleton, 1960).

Estudios clásicos sobre la función de este tipo de acusaciones en la fisión de linajes matrilineales se han realizado entre los cewa (Marwick, 1952, 1963, 1965, 1966, 1967), los yao (Mitchell, 1956) y los ndembu (Turner, 1957).

\section{El trabajo de Marwick}

El trabajo de campo entre los cewa lo inició Marwick en 1946. Durante más de un año estudió su lengua y su cultura y empezó a tomar notas de casos de infortunio. Dos viajes en los veranos de 1948-49 y 1952-53 le permitieron completar su información hasta 194 casos.

\footnotetext{
${ }^{2}$ De las tesis de Wilson y Nadel me he ocupado de forma crítica en González Echevarría (1984: 75 y ss.), donde desarrollé por primera vez el análisis que ahora voy a aplicar.
} 


\section{perifèria}

Número 5, Diciembre 2006

www.periferia. name

En repetidas ocasiones (1952, 1966 [1961], 1965), Marwick se adscribe al método hipotético-deductivo. No trata de inducir generalizaciones, sino de presentar sus datos de forma que puedan servir como contrastación a hipótesis que otros autores que se han ocupado de brujería suscriben, al menos implícitamente.

Estas hipótesis, reformuladas con algunas variantes a lo largo de sus escritos, son, en esencia, las siguientes:

1) Las creencias en brujería proporcionan una teoría de la causación.

2) Las creencias proporcionan una salida a la hostilidad, la frustración y la ansiedad.

3) Las creencias sirven para formular las tensiones que se dan en algunas relaciones sociales y para reformular o cortar estas relaciones. $Y$ esto en un doble aspecto: a) las acusaciones son un índice de tensiones entre acusador y acusado; y b) las informaciones que proporciona la gente cuando habla en abstracto de brujería, aunque no coinciden con la práctica de las acusaciones, dan a conocer su apreciación de las tensiones sociales.

4) Las creencias sirven de soporte a los valores sociales, porque indican el tipo de comportamiento que puede dar lugar a una acusación y porque, al trazar la imagen del brujo, definen dramáticamente lo que es considerado malo en cada cultura (Marwick, 1952: 120 y ss.; 1966: 1971.).

De la segunda hipótesis, que es la de Kluckhohn (1972 [1944]), se desembaraza Marwick con prontitud. Son las (1), (3) y (4) las que quiere someter a contrastación a través de la etnografía cewa. Para hacerlo desarrolla con más detalle la hipótesis tercera.

Tres son, para este autor, los factores que determinan la tensión en una relación social, identificada con una relación de competencia:

V: El valor conferido al objetivo por el que se compite.

P: La medida en que la relación envuelve a toda la personalidad.

C: La medida en que la relación está circunscrita tradicionalmente, por ejemplo, por normas legítimas tales como las que sostienen la distancia 


\section{perifèria}

Número 5, Diciembre 2006

www.periferia. name

social. En este sentido los estatus adscritos son fuente de mucha menos tensión, mientras que si el acceso a un estatus está más libre de determinaciones sociales generará muchos más conflictos.

Por otra parte, la tensión será expresada en acusaciones de brujería y hechicería si: a) hacerlo es congruente con las creencias tradicionales, b) hay suficiente material bruto, en forma de desgracias, para pensar en ataques de brujas y hechiceros; c) no existen o no se prefieren medios alternativos de expresar tensiones (1966: 173).

Más concretamente, y acercándose ya a lo que se ha llamado hipótesis obstétrica:

Las acusaciones de brujería, como ejemplos de conflicto social, tienen la función, inter alia, de formular tensiones de modo que permitan la ruptura de relaciones personales estrechas que no pueden ser cancelas y que no se pueden seguir manteniendo porque resultan insoportables (ibídem).

\section{Dificultades para la puesta a prueba de las hipótesis}

Antes de evaluar la contrastación de hipótesis de Marwick, es necesario bosquejar brevemente los aspectos de la organización social y de las creencias cewa que son relevantes para las acusaciones de brujería.

Los cewa del norte de Rodesia son cultivadores de azada, entre los que la ganadería tiene un interés creciente debido al éxito de la lucha contra la mosca tsétsé. Son matrilineales; con matrimonio inicialmente virilocal, al cabo de un tiempo la pareja se instala en el poblado de la esposa. Esos poblados tienen entre 40 y 200 chozas y a veces están divididos en secciones. Los poblados, o las secciones de un poblado, están ocupados por matrilinajes, khol. Dentro del khol se distinguen segmentos mawele (sing. bole) ordenados por la edad de sus cabezas. Cuando un linaje se hace inmanejable, se rompe en segmentos. 


\section{perifèria}

Número 5, Diciembre 2006

www.periferia.name

Los miembros matrilineales de la sección son usualmente un hombre, el jefe, con sus hermanas, los hijos de sus hermanas, los hijos de las hijas de sus hermanas, etc. Viven con él además sus propios hijos. El jefe del poblado es el de la sección del linaje fundador.

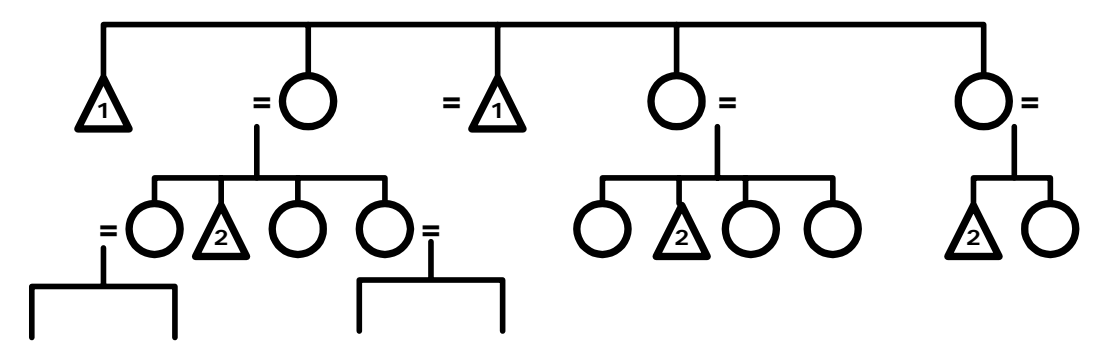

I lustración 1. Posibles jefes del matrilinaje en cada generación

La norma de sucesión a la jefatura es de hermano mayor a hermano menor. Cuando muere el último de los hermanos, el heredero es el hijo mayor de la hermana mayor, pero puede sustituirlo otro hombre de la generación segunda (un primo paralelo uterino) si éste demuestra su capacidad y descalifica al heredero. Este margen para la acción personal, unido a la valoración de la jefatura y a las intensas relaciones entre primos uterinos, hace de la competición por el cargo una fuente de tensión.

Los cewa creen en un Ser Supremo, en espíritus del linaje (los del hermano de la madre y el hermano de la madre de la madre), en un complejo de fuerzas sobrenaturales impersonales y en el poder de la magia. 


\section{perifèria}

Número 5, Diciembre 2006

www.periferia. name

A todos los que hacen mal por medios místicos los llaman nfiti. Se trata de personas necrófagas, que atacan a sus parientes matrilineales mediante el uso de medicinas, poseen familiares y transmiten su arte dentro de la familia, enseñándolo a un hijo o a un nieto. De los nfiti dicen los cewa que son mayoritariamente mujeres.

A veces distinguen dos tipos de nfiti : los que actúan por odio y aquellos a los que mueve el deseo de carne (Marwick, 1965: 73-79). Yo hablaré en general de brujas y brujería, porque la naturaleza del poder del nfiti es en este contexto irrelevante.

Dicen los cewa que la brujería se usa sobre todo en el interior del linaje, aunque no entre hermanos y en relación con el acceso a la jefatura o en disputas por propiedad, especialmente de ganado, que en los años cincuenta, en los que Marwick hizo su trabajo de campo, acababa de introducirse. También actúa la brujería entre co-esposas y entre gente que trabaja para el mismo patrón. De hecho Marwick contrasta a la vez las hipótesis teóricas de los antropólogos y las hipótesis folk de los cewa. Y lo hace por medio de la información sobre 194 casos de infortunio que consiguió recoger a lo largo de su investigación.

A) Hipótesis (1): Las creencias en la brujería proporcionan una teoría de la causación.

En el caso cewa, la explicación del infortunio en los episodios para los que hay información suficiente, es la siguiente (Marwick, 1965: 16): 


\section{perifèria}

Número 5, Diciembre 2006

www.periferia. name

1) Causas naturales ("Actos de Dios")

2) Actos de personas que no son brujos

- venganza por brujería

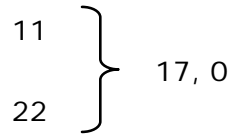

la propiedad o anti-adulterio, suicidios.

- otros actos, como ruptura de tabúes, uso de magia protectora de

3) Actos de brujos

- Brujos muertos por su propia brujería o en enfrentamientos con otros brujos

- Ataques de brujos contra no brujos

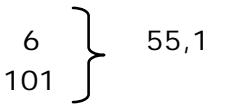

4) Actos de espíritus

$5 \quad 2,6$

Tabla 1. Explicaciones de los infortunios $(N=194)$

Del total de 194 casos, 118 implican creencias en brujería. Sin embargo, las desgracias que se deben a la actuación del brujo $(55,1 \%)$, o se relacionan con brujería $(60,8 \%)$, no responden a las afirmaciones de los cewa, que atribuyen a brujería "casi todos" los infortunios. Marwick se limita a constatar esta discrepancia entre las declaraciones generalistas y los hechos, discrepancia que se repite a propósito del sexo de los brujos pues, lejos de ser "casi todos mujeres", en los cien casos en los que el sexo del brujo se conocía, 58 eran hombres y mujeres 42 (Marwick, 1965: 103).

B) Hipótesis (4): Las creencias de brujería sirven de soporte a los valores morales.

Las informaciones relativas a los casos de desgracia muestran también que las creencias de brujería sirven de soporte a las normas morales, y no tanto por las características de los brujos, a los que se atribuyen rasgos antisociales en la mitad de los casos, como por las relaciones que se establecen entre las desgracias y el comportamiento moral de las víctimas y sus allegados. Así, en los 101 casos de brujos que atacan a los no brujos, 49 de los acusados tienen características reprochables: 17 son ya brujos conocidos; 9, celosos o avaros; 7, violentos y 


\section{perifèria}

Número 5, Diciembre 2006

www.periferia. name

pendencieros; 5 están envueltos en actividades siniestras, como incesto y magia de caza; 4 son impotentes o estériles y 7 tienen otras características negativas (Marwick, 1965: 239).

En cuanto a las víctimas, en los 118 casos en que la desgracia tenía relación con brujería, 47 carecían de implicaciones morales, 56 tenían que ver con faltas de la propia víctima, y 15 con faltas de allegados. Para los 76 casos restantes, en los que las causas del infortunio eran naturales o se debían a los actos de no brujos o de espíritus estas cifras eran, respectivamente, 30, 27 y 19 (o.c.: 16). Es decir, un total de 117 desgracias se vinculaban con transgresiones de las normas morales por parte de las víctimas o sus allegados y solo 77 carecían de estas implicaciones. Marwick ha recogido y comentado (o.c.: 227 y ss.) las transgresiones más frecuentes, que son: incumplimiento de obligaciones tradicionales ( 23 casos), fallos en el ritual o ruptura de tabúes (10), promiscuidad sexual (17), celos sexuales y adulterio (7), ser brujo (17), excesivamente rico (12) o ambicioso (5), agresivo (10), o ladrón (10) y, finalmente, poco viril e insociable (6).

Los datos parece que, efectivamente, corroboran las hipótesis (1) y (4); veamos ahora qué sucede con la hipótesis tercera.

C) Hipótesis (3): Las creencias en brujería sirven para formular las tensiones que se dan en algunas relaciones sociales y para reformular o cortar estas relaciones.

El análisis de los datos es especialmente importante para corroborar o refutar la tesis obstétrica: si efectivamente las acusaciones de brujería son un índice de las relaciones sociales tensas, si la más tensa entre los cewa es entre posibles competidores por la jefatura del matrilinaje, y si además una de las funciones de las acusaciones es permitir que se rompan relaciones personales estrechas que se han hecho insoportables, cabría esperar un máximo de acusaciones entre gente de distintos segmentos del mismo linaje, especialmente entre los competidores directos, esto es primos paralelos matrilaterales.

¿Y qué dicen los datos? Muy poco. Tanto en el artículo de 1961 (citado 1966) como 


\section{perifèria}

Número 5, Diciembre 2006

www.periferia. name

en Sorcery in its social setting (1965), Marwick proporciona varias tablas donde se recogen las relaciones de parentesco y espaciales entre acusado y víctima, acusado y acusador, acusador y víctima (1966: 167; 1965, especialmente 152-153). Los números para acusador, acusado y víctima no coinciden con el número de casos, 101, porque 1을 en sólo 79 hay información sobre la identidad de los acusadores, y $2^{\circ}$ ) en varios de los casos, acusado y/o víctima y/o acusador son más de uno.

Voy a recoger sólo una tabla, donde se dan los porcentajes de lazos entre 1) brujo y víctima, 2) acusador y brujo, y 3) acusador y víctima, distribuidos por categorías de relaciones (Marwick, 1965: 152):

\begin{tabular}{|c|c|c|c|}
\hline Tipo de relación social & $\begin{array}{l}\text { 1) Brujo y víctima } \\
(N=115)\end{array}$ & $\begin{array}{l}\text { 2) Acusador y brujo } \\
\text { ( } N=112)\end{array}$ & $\begin{array}{l}\text { 3) Acusador y víctima } \\
(\mathrm{N}=90)\end{array}$ \\
\hline $\begin{array}{l}\text { (a) Parentesco matrilineal } \\
\text { Igual segmento } \\
\text { Distinto segmento } \\
\text { Segmento no identificado }\end{array}$ & $\begin{array}{l}17,4 \\
29,6 \\
11,3 \\
58,3\end{array}$ & $\begin{array}{c}13,4 \\
33,0 \\
3,6 \\
50,0\end{array}$ & $\begin{array}{l}33,3 \\
22,2 \\
22,2 \\
77,7\end{array}$ \\
\hline $\begin{array}{l}\text { (b) Parentesco no matrilineal } \\
\text { Esposas } \\
\text { Coesposas, reales o potenciales } \\
\text { Otros afines } \\
\text { Otros } \\
\end{array}$ & $\begin{array}{c}5,2 \\
1,7 \\
11,3 \\
13,9 \\
32,1\end{array}$ & $\begin{array}{c}1,8 \\
0,9 \\
31,2 \\
7,1 \\
41,0\end{array}$ & $\begin{array}{c}4,4 \\
- \\
8,9 \\
7,8 \\
21,1\end{array}$ \\
\hline (c) No pariente & 8,7 & 8,9 & 1,1 \\
\hline (d) Relación desconocida & 0,9 & - & - \\
\hline
\end{tabular}

Tabla 2. Distribución porcentual de relaciones

En relación con la función obstétrica de las acusaciones de brujería, con su papel de instrumento para superar las fidelidades del parentesco matrilineal y hacer posible la ruptura del linaje, es muy poco lo que podemos inferir de estos datos o, si aceptamos las declaraciones de principio de Marwick, lo que estos datos pueden corroborar. Porque si bien es cierto que acusador y brujo pertenecen a distintos segmentos del linaje más del doble de veces que el mismo segmento (33\% y $13,4 \%$ respectivamente), también lo es que en la mitad de los casos, acusador y 


\section{perifèria}

Número 5, Diciembre 2006

www.periferia. name

acusado no pertenecen al mismo matrilinaje. En definitiva, sólo un tercio del total de acusaciones se da entre personas que pueden estar en la situación que se ha supuesto más conflictiva. Y subrayo pueden, porque del hecho de que dos personas pertenezcan a distintos segmentos de un matrilinaje no se deduce que estén en situación de competir por el puesto de jefe. $Y$ en los casos que se recogen en Marwick (1952) así se indica.

Pero aun limitándose al matrilinaje, un 33\% de acusaciones entre segmentos frente a un $13,4 \%$ en el interior del mismo segmento no significa por sí solo que las tensiones entre segmentos sean más fuertes que en su interior. Si un hombre tiene un hermano y tres primos paralelos matrilaterales, para que hubiese un indicio de tensión mayor con sus primos que con sus hermanos, los enfrentamientos con aquéllos tendrían que ser más de tres veces mas frecuentes que con el hermano, a no ser que la relación entre primos sea menos frecuente.

Marwick es consciente de esta debilidad, de que tendría que haber recogido no tasas brutas sino frecuencias relativas al volumen de interacción. Lo es ya en 1961, aunque intenta una aproximación tangencial comparando los porcentajes de las relaciones entre acusador y víctima, y acusador y brujo en el interior de cada categoría de parentesco (p. 181). Pero tampoco estos datos discriminan el conflicto en las relaciones entre hermanos y las relaciones entre primos porque el hecho de que el hermano acuda al adivino para averiguar la causa de la desgracia no es indicador de hostilidad entre primos, sino una de las obligaciones de los parientes próximos. A no ser que aquí se esté llamando hostilidad a la distancia genealógica.

En 1965 sigue confiriendo significación a lo que llama balance entre "hostilidad" y “afiliación”, cada vez con menos entusiasmo. En 1967, claramente liberado ya de la obligación de escribir «su monografía» sobre los cewa, admite:

Dentro de cada categoría de relación hay que expresar las acusaciones de brujería como proporciones del volumen total de interacción que caracteriza a dicha relación antes de que se puedan hacer comparaciones válidas. El 


\section{perifèria}

Número 5, Diciembre 2006

www.periferia. name

número de personas con las que Ego mantiene una relación particular no es más que una de las bases para estimar el universo de interacción social característico de la categoría de relación concernida. Otra puede ser la cantidad de tiempo que pasa con personas con las que está en una relación determinada, y otra, la medida en que esta interacción con ellos, frente a la que tiene, por ejemplo con sus propios hermanos, está pautada por nociones de etiqueta, evitación y familiaridad (Marwick, 1967: 243).

Pienso que esas últimas líneas de Marwick, las que corresponden en cierto modo a su autocrítica, son el aspecto más relevante, desde el punto de vista metodológico, de su tesis obstétrica. Es verdad que su propia conclusión vuelve a ser holística, porque supone que el trabajador de campo en una sociedad basada en el parentesco debería de recoger todos los datos genealógicos y demográficos que conciernen a personas envueltas en supuestos ejemplos de brujería, de manera que los datos sirvan para estimar el universo de interacción que se debe construir. Marwick no parece tener presente que «todos» los datos sólo pueden ser recogidos a partir de un diseño de investigación que otorgue significación a los datos en función de las hipótesis que queremos poner a prueba, y que sin este diseño el trabajo de campo no proporcionará materiales adecuados para la contrastación.

El discurso de Marwick no es tan hipotético-deductivo como sostiene, porque aunque iniciara el trabajo de campo atento a las creencias en brujería como indicadores de tensión, su papel en la ruptura del linaje no parece que se convierta en hipótesis hasta que las afirmaciones de los propios cewa le ponen sobre la pista, en este sentido es emergente avant la letre. De hecho, en 1952, lo que afirma es que: a) "se toma las creencias en brujería como índice de la tensión social"; b) "se las considera como fuerzas que soportan el sistema de valores, y en consecuencia la estructura social".

Pero aun si se tienen claras las hipótesis, no basta. Hay que establecer formas de correspondencia entre teoría y datos que establezcan el tipo de informaciones que tenemos que buscar. Si una de las hipótesis que se va a poner a prueba es que las 


\section{perifèria}

Número 5, Diciembre 2006

www.periferia. name

creencias en brujería son índice de la tensión social y se supone, como hace Marwick, que la tensión (en este caso en una relación de competencia), es: a) directamente proporcional a la importancia que se confiere al objeto por el que se compite (V); b) directamente proporcional a la medida en que la relación envuelve a toda la personalidad, (P) y c) inversamente proporcional a la medida en que la relación social está tradicionalmente pautada $(C)$; tenemos que arbitrar definiciones operacionales que nos permitan medir $\mathrm{V}, \mathrm{P}$ y $\mathrm{C}$.

Sólo mediante el establecimiento de indicadores para medir V, P, C, podemos decir si la relación entre co-esposas es más tensa o menos tensa que la relación entre primos paralelos matrilaterales y si existe una incidencia correlativa (incidencia medida por procedimientos que también deben especificarse) de acusaciones de brujería, hecho que iría en apoyo de la hipótesis de que las creencias en brujería sirven para formular tensiones sociales.

Y lo que quizás sea más importante. Si los antropólogos se propusieran seriamente contrastar sus hipótesis, serían más cautelosos a la hora de formular explicaciones - o si se prefiere interpretaciones- en términos de la "medida en que toda la personalidad está envuelta en una relación social" o en términos de "la unidad del grupo de hermanos". Nunca se insistirá bastante en el riesgo que se corre en las ciencias de la cultura cuando se utilizan como términos teóricos conceptos con cuyo uso preteórico estamos familiarizados (un ejemplo notable es «matrimonio») sin que se dé una caracterización adecuada del uso teórico de dichos términos dentro de la teoría cuya formulación los incluye.

Volviendo a las hipótesis de Marwick: si son las propias acusaciones de brujería las que se toman como indicadores de tensión, entonces una relación será más tensa que otra si la incidencia - de nuevo "adecuadamente medida" - de las acusaciones de brujería es mayor, pero en este caso sería la tensión así definida la que hay que explicar. Y si es la «imagen del brujo» la que se toma como indicador de tensión (sería la hipótesis 3b) --en el caso cewa la afirmación nativa de que los nfiti son mayoritariamente mujeres-, hay que explicar la discrepancia entre la teoría 


\section{perifèria}

Número 5, Diciembre 2006

www.periferia. name

indígena y las acusaciones reales por una vía que no sea invocar «proyecciones» psíquicas no corroborables.

En aquel artículo de 1952 Marwick concluía que las creencias en brujería proporcionan un medio de romper relaciones sociales que se han esclerotizado o se han hecho demasiado insistentes y que incluso pueden ser el único medio de romper o alterar fundamentalmente una relación cuando ha llegado a hacerse insoportablemente tensa y cuando no hay ningún modo socialmente aprobado de hacerlo. Es esta conclusión, convertida en hipótesis como ya he recogido, que haría de las acusaciones de brujería un medio para cancelar relaciones estrechas que se han hecho insoportables (1966: 173), la que no puede poner a prueba con los mismos datos que la habían sugerido. Éste es el problema con el que se bate Marwick en 1963, 1965, 1966 y al que finalmente, en 1967, se rinde. El trabajo de campo, o es puesta a prueba de hipótesis formuladas previamente, y debe responder a un proyecto diseñado con este fin, o es la fuente de nuevas hipótesis que deben de ser a su vez corroboradas o refutadas en contraste con nuevos datos. También puede ser las dos cosas. Merton ha subrayado bien el elemento de serendipity, de posibilidad de hacer descubrimientos inesperados de forma accidental, al que debe de estar abierta toda investigación empírica (1972: 113); Agar ha basado en las quiebras su metodología y hoy todos estaríamos de acuerdo en el carácter emergente de mucha de la investigación etnográfica ${ }^{3}$. Pero aunque la etnografía sirva a la vez para generar hipótesis, para ponerlas a prueba, y para modificarlas y clarificarlas, conviene no confundir los distintos trámites, porque tienen exigencias distintas.

\section{Bibliografía}

Agar, M. 1992 [1982], “Hacia un lenguaje etnográfico", en C. Reynoso, comp. El surgimiento de la Antropología postmoderna. Barcelona: Gedisa. 779-795.

\footnotetext{
${ }^{3}$ No de toda ni de todas sus fases. Cuando el invocado carácter emergente de una investigación sirve de excusa para no explicitar las hipótesis, supuestos no problemáticos y conceptos previos que la informan se rehuye el primer trámite de crítica no empírica. Se produce así una curiosa paradoja: los prediseños emergentes se parecen a los hipotético-deductivos en la subestimación del contexto del descubrimiento.
} 


\section{perifèria}

Número 5, Diciembre 2006

www.periferia. name

Douglas, M. (1976). "Brujería: el estado actual de la cuestión”, en M. Gluckman y otros. Ciencia y brujería. 31-72. Barcelona: Anagrama

Evans-Pritchard,E.E. (1978 [1937]). Brujería, magia y oráculos entre los Azande. Barcelona. Anagrama.

Feyerabend, P.K. (1974). Contra el método. Barcelona. Ariel.

Fortes, M. (1975 [1951]). "La estructura de los grupos de filiación unilineal", en L. Dumont. Introducción a dos teorías de la Antropología Social: 170-198. Barcelona. Anagrama.

Gadamer, H.G, (1993 [1960]), Verdad y Método I. Barcelona: Sígueme.

González Echevarría, A. (1984). Invención y castigo del brujo en el Africa Negra. Teorías sobre la brujería. Barcelona: Ediciones del Serbal.

- (1987). La construcción teórica en Antropología. Barcelona: Anthropos.

Jarvie, I.C. (1967 [1964]). The Revolution in Anthropology. Londres: Routledge and Kegan Paul.

Kluckhohn, C. (1972 [1944]). Navaho Witchcraft. Boston: Beacon Press.

Marwick, M.G. (1952). "The social context of Cewa witch beliefs". Africa. XXII, 2: 120-135 у 3: $100-112$.

- (1963). "The sociology of sorcery in a Central African Tribe". African Studies, 22, 1: 1-21.

- (1965). Sorcery in its social setting. A study of the Northern Rhodesian Cewa. Manchester: Manchester U.P.

- (1966 [1961]). "Some problems in the sociology of sorcery and witchcraft", en Fortes y Dieterlen, eds. African Systems of Tought. Londres. Oxford U.P.: 171191.

- (1967). "The study of witchcraft", en A.L. Epstein, ed. The Craft of Social Anthropology. Londres: Tavistock: 231-244.

Merton, T. (1972). Teoría y estructura sociales. México. F.C.E.

Middleton, J. (1960). Lugbara Religion. Londres. Oxford U.P.

Mitchell, J.C. (1956). The Yao Village. A Study in the social structure of a Malavien People. Manchester. University of Manchester Press.

Murdock, G.P. (1957). "World Ethnographic Sample”, American Anthropologist. N.S. 59, 4:664-687.

- (1975 [1957]). “World Ethnographic Sample”, American Anthropologist, 59 pp. 664-687 (y en Llobera, ed. [1975], La Antropología como ciencia.

Barcelona: Anagrama. pp. 203-230.

Nadel, S.F. (1952). "Witchcroft in four African Societies: An Essay in comparison". American Anthropologist 54 (1): 18-29. 


\section{perifèria}

Número 5, Diciembre 2006

www.periferia. name

- (1970) Nupe Religion. New York: Schocken Books.

San Román, T. (1989). "Sobre el objeto y el método en Antropología", Quaderns de I'Institut Catalá d'Antropología, no 5: 122-133.

- (1996). “De la intuición a la contrastación. El trabajo de campo en Antropología y en la formación de los nuevos antropólogos", en González Echevarría, A. coord. Epistemología y Método, Actas del VII Congreso de Antropología Social. Zaragoza: Instituto Aragonés de Antropología/Federación de Asociaciones de Antropología del Estado Español

Turner, V. (1957). Schism and continuity in a African Society. A Study of Ndembu Village Life. Manchester: University of Manchester Press.

Wilson, M. (1951). "Witch belief and social structure". American Journal of Sociology, vol. LVI, 4: 307-313.

- (1970 [1950]). Good Company. A study of Nyakyusa Age Villages. Boston: Beacon Press

Winch. P. (1976[1958]). The Idea of a Social Science and its Relations to Philosophie. Londres: Routledge and Kegan Paul. 